\title{
Thermodynamics of Concanavalin A Self-Association in the Presence of Osmolytes
}

\author{
Tyler Pfister*, Shamus Cooper, \& Jeffrey K. Myers \\ Department of Chemistry, Davidson College, NC \\ Students:Typfister17@alumni.davidson.edu*,Shcooper16@alumni.davidson.edu \\ Mentor:Jemyers@davidson.edu
}

\begin{abstract}
Protein-protein interactions are critical for biological function and depend significantly on environmental factors. A wide variety of small organic molecules that comprise the cellular environment are capable of interacting with proteins to affect folding, binding, and association. The plant lectin concanavalin A (ConA) undergoes a reversible, $\mathrm{pH}$ dependent dimer-to-tetramer equilibrium and has been used in our laboratory as a model system to study the effect of osmolytes on protein self-association. Previous research determined that trimethylamine $N$-oxide (TMAO) stabilizes the tetrameric conformation, while urea favors the dimer. Studying the equilibrium over a range of temperatures allowed quantification of the enthalpy change $(\Delta \mathrm{H})$ and entropy change $(\Delta \mathrm{S})$ of tetramer formation. Urea increased both the $\Delta \mathrm{H}$ and $\Delta \mathrm{S}$ of tetramer formation, while TMAO decreased both. These effects are consistent with preferential hydration of the dimer-dimer interface in TMAO solution and preferential binding of urea to the interface.
\end{abstract}

\section{KEYWORDS}

Concanavalin A; Osmolytes; Trimethylamine N-oxide; Urea; Protein-Protein Interaction; Equilibrium; Enthalpy; Preferential Hydration

\section{INTRODUCTION}

Many proteins in biological contexts are known to exist as oligomers; therefore it is necessary to study the process of protein self association to gain a full picture of protein folding. The conformational changes involved in protein folding are sensitive to environmental conditions including $\mathrm{pH}$, temperature, and possible interactions with small molecules. Osmolytes are a class of small organic molecules that accumulate naturally in cells where they function to mitigate osmotic stress. ${ }^{1}$ Osmolytes have been characterized in detail with respect to their ability to alter the thermodynamics of monomeric protein folding. ${ }^{2-7}$ However, relatively few studies have addressed the effect of osmolytes on protein-protein interactions. ${ }^{8-10}$

Most osmolytes stabilize protein structure, and are thought to be preferentially excluded from the peptide backbone, causing the protein to favor a folded state where there is less surface area to interact with the osmolyte., 5, 11, 12 Additionally, osmolytes are able to sequester water molecules, resulting in a local hydrophobic environment that favors the folded protein. Consequently, the melting temperature $\left(\mathrm{T}_{\mathrm{m}}\right)$ and the free energy of unfolding $(\Delta \mathrm{G})$ are elevated in the presence of stabilizing osmoltyes. ${ }^{4},{ }^{11}$ Evidence also supports osmolytes' role in structuring regions of proteins that are intrinsically disordered. This may allow for molecular recognition and ligand binding when these regions are stabilized in correct conformations. It has been suggested that diseases like Alzheimer's and Parkinson's that involve formation of amyloid fibrils might result in part from osmolyte stabilization of certain oligomeric precursors. ${ }^{12}$

Destabilizing osmolytes are thought to interact favorably with the peptide backbone through hydrogen bonding, thus favoring unfolding. Destabilizing osmolytes pose their own threat to protein function since moderate concentrations of compounds like urea (which accumulates in cartilaginous fish) ${ }^{13,14}$ lead to destabilization of proteins. Lin et al. found that trimethylamine $N$-oxide (TMAO), a stabilizing osmolyte, can help combat the denaturation effects of urea. They determined that urea preferentially bound to the denatured form of the protein, while TMAO was preferentially excluded. This results in a change in the $\Delta \mathrm{G}$; urea decreases the $\Delta \mathrm{G}$ resulting in a smaller $\mathrm{T}_{\mathrm{m}}$. TMAO increased $\Delta \mathrm{G}$, resulting in a larger $\mathrm{T}_{\mathrm{m}}$. In combination, the net effect was the algebraic sum of their individual effects. ${ }^{13}$ Wang and Bolen reached a similar conclusion by using a transfer model to measure the transfer free energy change of proteins and individual amino acids. ${ }^{14}$ This allowed them to quantify the transfer free energy of the side chains of each amino acid. From their results, it was concluded that TMAO preferentially interacts with the backbone to destabilize the denatured state. Mello and Barrick, who showed that free energy transitions varied linearly with TMAO concentration, further validated these results..$^{15}$ 
Despite the prevalence of research concerning the influence of osmolytes on protein denaturation, there is considerably less research on their effect on protein-protein interactions. The binding of protein monomers to each other is an inherent part of the folding of oligomeric proteins, and can be viewed as a special case of a macromolecule binding to its ligand. The ability of osmolytes to preferentially interact with water or protein may have an effect on the change in free energy of binding. ${ }^{16}$ This effect is due in part to a hydration effect and not the result of crowding. When the binding of a TATA-box binding protein to its $20 \mathrm{bp}$ DNA promoter was examined with betaine, sucrose, and triethylene glycol (TEG), there was no change in the dissociation constant $\left(\mathrm{K}_{\mathrm{d}}\right)$ in the presence of TEG, but betaine and sucrose decreased the $\mathrm{K}_{\mathrm{d}} 18$ and 5-fold respectively. This results from the increased number of water molecules released upon binding and concomitant entropy change. ${ }^{16,17}$ Alternately, osmolytes can decrease the binding affinity through increased water uptake used to mediate the ligand binding. One example is the binding of lysozyme to a monoclonal antibody, which shows a 26 -fold binding affinity decrease in the presence of betaine. ${ }^{18}$ Additionally, osmolytes can preferentially interact with ligands to change the free energy of binding. ${ }^{16}$

Previous work in our laboratory has established Concanavalin A (ConA) as a convenient model system in which to study the effects of osmolytes on protein self-association. ${ }^{10}$ Concanavalin A is a commercially available plant lectin from jack bean, Canavalia ensiformis. Its chemical and physical properties have been extensively characterized in earlier works. ${ }^{19}{ }^{20} \mathrm{ConA}$ is a metalloprotein that binds $\alpha$-D-mannopyranoside and $\beta$-D-glucopyranoside through the use of one manganese $\left(\mathrm{Mn}^{2+}\right)$ and one calcium $\left(\mathrm{Ca}^{2+}\right)$ per subunit. ${ }^{21-25}$ This unique functionality has lead to its use in analytical separation of glycosylated proteins and mitogen stimulation of the immune system. The secondary structure of the protein consists of two large anti-parallel $\beta$-sheets. A twisted sheet of seven strands passes through the center and the back is formed by a bowed six-strand sheet. ${ }^{21-23}$ ConA can be found as a tetramer or dimer under cellular conditions, however the tetramer predominates at neutral $\mathrm{pH}$. The structure can be described as a dimer of dimers with a center water-filled cavity and a relatively large interface, the surface area of which is estimated to be $1150 \AA^{2}$ per dimer. ${ }^{10}$ The equilibrium has been studied previously using sedimentation equilibrium in Senear and Teller. They determined that protonation of a single Histidine residue at $\mathrm{pH}$ 6.55, likely His 51 or His 121, leads to destabilization of the tetramer. ${ }^{26}$

ConA is a convenient model because it allows for study of the equilibrium by titrating samples to change the $\mathrm{pH}$ and monitoring the reversible dimer to tetramer process through circular dichroism (CD). Near UV CD proved useful in monitoring the equilibrium due to small changes in the environment surrounding aromatic side chains upon tetramer formation. It was found that osmolytes that are known to stabilize monomeric protein folding also favor formation of the ConA tetramer. Urea, a destabilizing osmolyte, favored the dimeric form of ConA. However, theoretical models did not reliably predict the effects of osmolytes on ConA association in a quantitative fashion. Thus, further research is needed to fully understand the details of osmolyte interactions with proteins.

In this work, the temperature dependence of ConA association in the presence of TMAO and urea was analyzed to determine the effect of these osmolytes on the enthalpy and entropy changes that accompany association.

\section{METHODS AND PROCEDURES}

ConA preparation.

ConA, lyophilized from Canavalia ensiformis (jack bean), was purchased from Sigma and found to contain a significant amount of hydrolyzed subunits. Purification was described previously ${ }^{10,26}$ and proceeded by preparing a $10 \mathrm{mg} / \mathrm{mL}$ solution of ConA in 5 $\mathrm{mM}$ sodium acetate buffer $(\mathrm{pH}=2.3)$, which was then dialyzed against $1 \%$ ammonium bicarbonate $(\mathrm{pH}=7.0)$ for $10-12$ hours at $37^{\circ} \mathrm{C}$. Dialysis was followed by centrifugation at 25,000 $\mathrm{g}$ for 20 minutes to remove precipitate. The supernatant was passed through a $0.22 \mu \mathrm{m}$ filter and dialyzed against ConA storage buffer $(50 \mathrm{mM}$ sodium phosphate pH $6.5,0.5 \mathrm{M} \mathrm{NaCl}, 0.2 \mathrm{mM} \mathrm{CaCl} 2$ and $\mathrm{MnCl}_{2}, 0.1 \mathrm{mM} \mathrm{NaN}_{3}$ ). Protein concentration was determined using $\mathrm{UV}$ absorbance spectroscopy at $280 \mathrm{~nm}$ using an extinction coefficient $E_{\mathrm{cm}}{ }^{1 \%}=11.4 .^{26}$

\section{Spectroscopic measurements of equilibrium}

All near-UV CD measurements were obtained on a Jasco J-815 spectropolarimeter using a $1.0 \mathrm{~cm}$ path length quartz cuvette with a bandwidth of $2.0 \mathrm{~nm}$ while temperature was maintained using a Peltier-type temperature controller (Quantum Northwest) under constant stirring.

Samples were prepared with $0.5 \mathrm{mg} / \mathrm{mL}$ ConA in titration buffer with or without $1.0 \mathrm{M}$ osmolytes. Titration buffer was made 5 times concentrated $(250 \mathrm{mM}$ acetate, $250 \mathrm{mM}$ phosphate, $0.5 \mathrm{M} \mathrm{NaCl}, \mathrm{pH}=7.5)$. TMAO was prepared at $4.0 \mathrm{M}$ in deionized water and washed with amberlite to remove contaminants. The resin was filtered away and concentration was determined using refractive index measurement. Urea was prepared at $6 \mathrm{M}$ in deionized water and the concentration was again determined by refractive index measurement. Samples were titrated from high $\mathrm{pH}$ to low with $6.05 \mathrm{M} \mathrm{HCl}$ using variable volumes while stirring. Separate titrations were conducted at different temperatures between $15-35^{\circ} \mathrm{C}$ using $5^{\circ} \mathrm{C}$ increments. At each pH point, $\mathrm{CD}$ signal 
was determined by taking the average of 30 two-second measurements at a wavelength of $284.0 \mathrm{~nm}$ and bandwidth of $2.0 \mathrm{~nm}$. Signals were corrected to account for dilution caused by adding $\mathrm{HCl}$ solution. The $\mathrm{pH}$ at each point was determined previously by a mock titration that used ConA storage buffer without protein mixed with titration buffer.

Data analysis.

The following equation was fit to the data from each titration:

$$
\text { Signal }=\frac{\left[m_{l} \times p H+b_{l}\right]+\left[m_{u} \times p H+b_{u}\right]}{1+10^{\left[n\left(p H-p H_{m i d}\right)\right]}}
$$

Equation 1.

where $m_{1}$ and $m_{u}$ are the slopes of the lower and upper baselines respectively, $b_{1}$ and $b_{u}$ are the intercepts of the lower and upper baselines respectively, $\mathrm{n}$ is the number of protons dissociated per dimer, and $\mathrm{pH}_{\mathrm{mid}}$ is the observed $\mathrm{pH}$ midpoint of transition. Senear and Teller previously determined that the equilibrium can be described using the following equation: ${ }^{26}$

$$
\ln \mathrm{K}_{\mathrm{app}}=\ln \mathrm{K}_{\mathrm{assoc}}-4 \ln \left(1+10^{-\mathrm{pH}} / 10^{-\mathrm{pK}_{\mathrm{a}}}\right)
$$

Equation 2.

where $K_{a p p}$ is the apparent association constant, $K_{a s s o c}$ is the association constant for deprotonated dimers and the pKa of the relevant histidine residue was determined to be 6.55 by Senear and Teller. ${ }^{26} \mathrm{~K}_{\text {app }}$ was determined to be $1.11 \times 10^{5}$ at the midpoint of the $\mathrm{pH}$ transition for the constant $0.50 \mathrm{mg} / \mathrm{mL}$ ConA concentration used. Van't Hoff plots were then constructed and the $\Delta H$ and $\Delta \mathrm{S}$ were determined from the slopes of fitted lines.

\section{RESULTS}

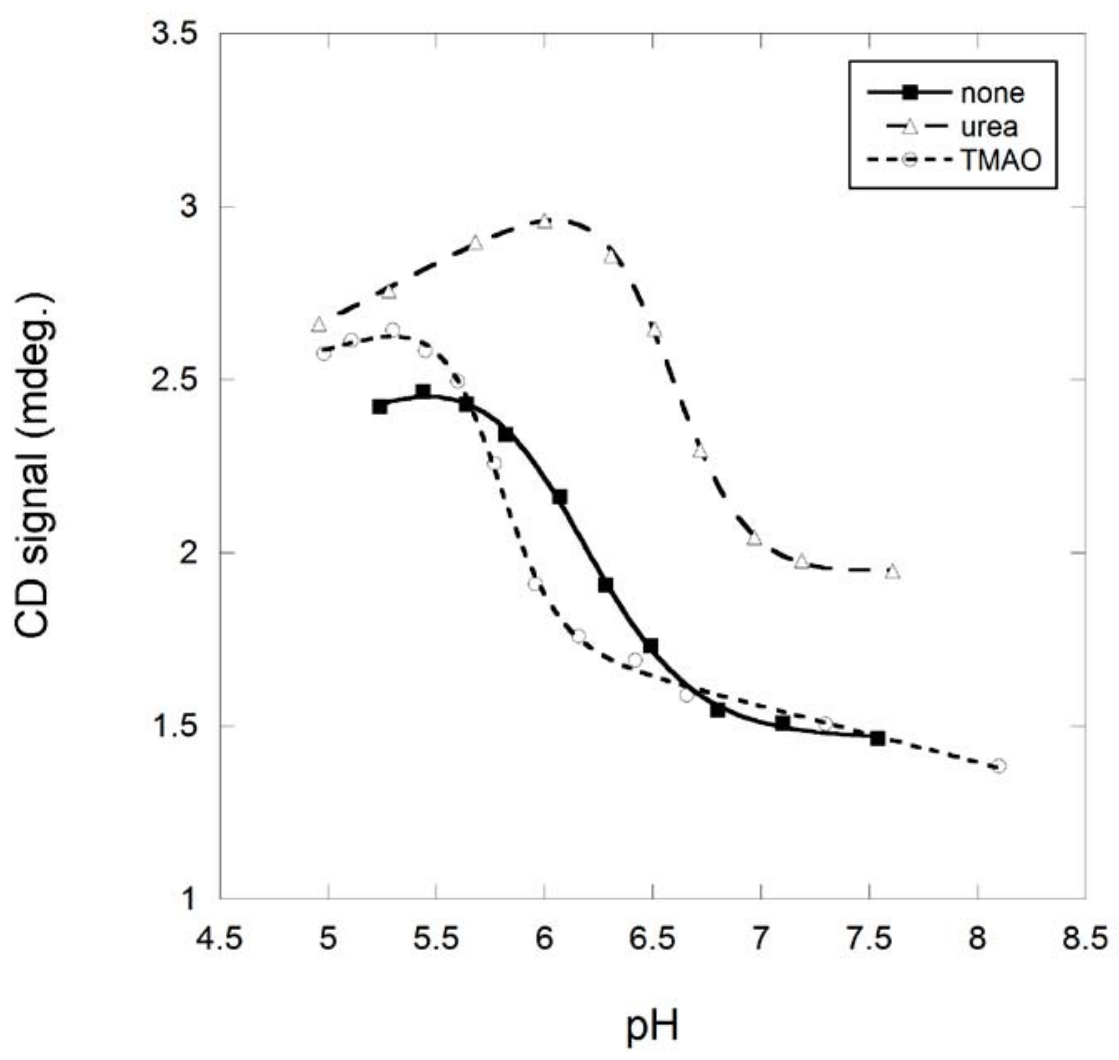

Figure 1. Titrations followed by circular dichroism. $\mathrm{pH}$ induced transitions from tetramer (neutral $\mathrm{pH}$ ) to dimer (acidic $\mathrm{pH}$ ) of ConA at $20^{\circ} \mathrm{C}$ followed by circular dichroism spectroscopy. Lines are fits to Equation 1 , used to determine the $\mathrm{pH}$ midpoint. 
Data from representative titrations are shown in Figure 1. The tetramer-to-dimer transition occurs when samples were titrated to low $\mathrm{pH}$ from a starting $\mathrm{pH}$ around 7.5. The initial and final baselines appear similar for titrations containing no osmolyte compared with those containing TMAO. This is not the case when ConA was titrated from pH 7.5 to 5.0 in $1.0 \mathrm{M}$ urea Figure 1. The addition of urea appears to affect the structure of the tetramer as evidenced by the change in high $\mathrm{pH}$ baseline. This is possibly due to destabilization of protein structure. An analogous effect is observed in the low pH baseline. Also notable is the fact that TMAO binds a proton with a $\mathrm{pKa}$ of 4.7, so that at the lower $\mathrm{pH}$ range of our experiments some of the TMAO is protonated; protonated TMAO might affect the equilibrium differently. ${ }^{27}$

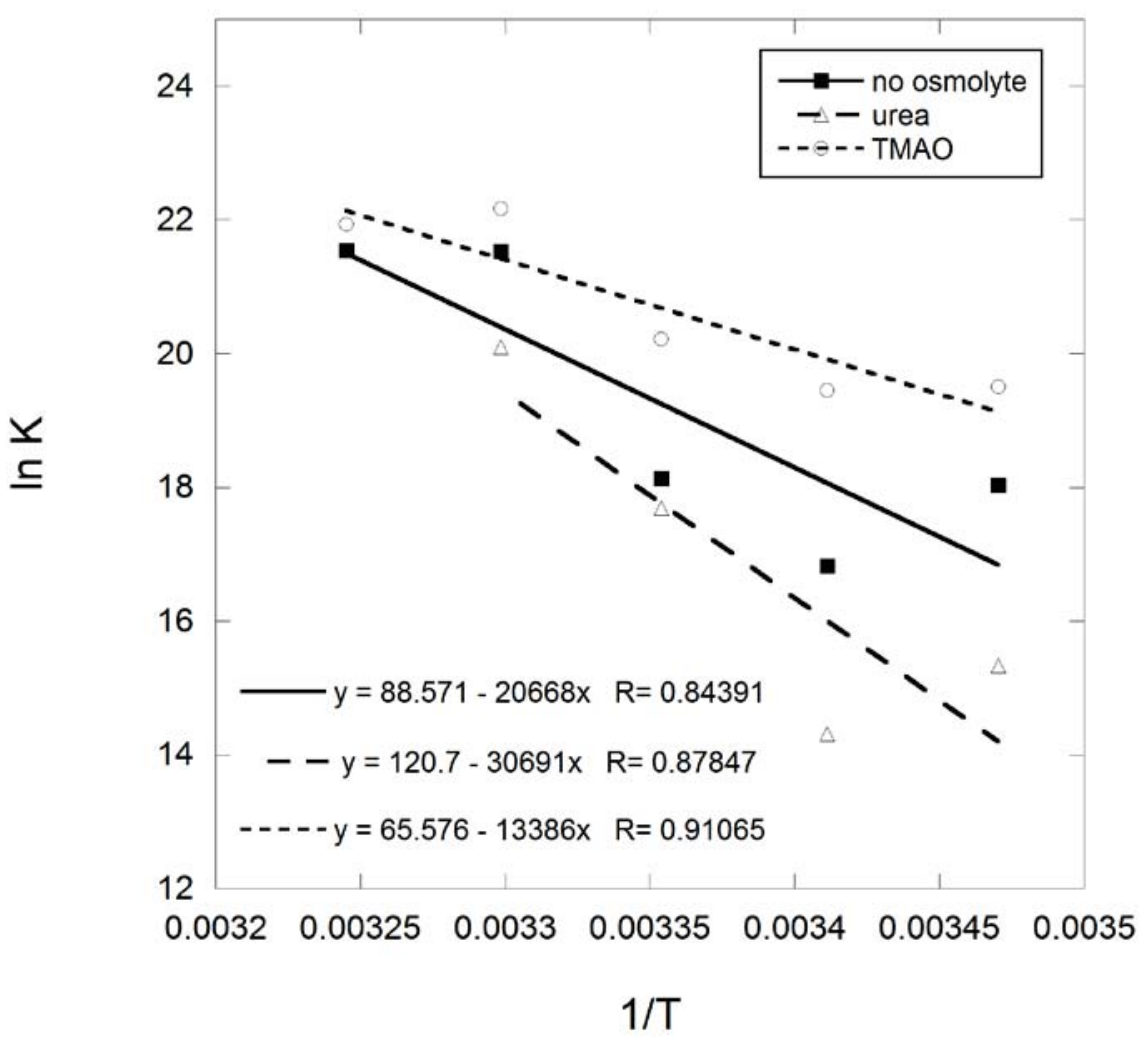

Figure 2. Van't Hoff plots for ConA association with TMAO, urea, and in the absence of osmolytes. Association constants were calculated using $\mathrm{pH}$ midpoint data from titrations at varying temperatures. Equations for linear fits are shown.

\begin{tabular}{|c|c|c|c|}
\hline Osmolyte & $\Delta \mathbf{G}_{298}(\mathrm{~kJ} / \mathrm{mol})$ & $\Delta \mathbf{H}_{298}(\mathrm{~kJ} / \mathbf{m o l})$ & $\Delta \mathbf{S}_{298}(\mathrm{~J} / \mathrm{mol} \cdot \mathbf{K})$ \\
\hline None & $-44.9 \pm 0.8$ & $171 \pm 63$ & $736 \pm 211$ \\
\hline Urea & $-41.9 \pm 0.8$ & $255 \pm 98$ & $1003 \pm 332$ \\
\hline TMAO & $-50.3 \pm 0.8$ & $111 \pm 29$ & $545 \pm 98$ \\
\hline
\end{tabular}

Table 1. Thermodynamic Data for ConA Association. Quantities for $\Delta \mathrm{H}$ and $\Delta \mathrm{S}( \pm$ s.e $)$ were determined from the lines of best fit in Figure 2. Free energy changes were calculated from $\Delta \mathrm{G}$ $=\Delta \mathrm{H}-\mathrm{T} \Delta \mathrm{S}$, and the error was assumed identical to Silvers and Myers (2013). ${ }^{10}$ 
In all cases, a sigmoidal shaped curve was obtained; these curves were fit to Equation 1 to provide the midpoint $\mathrm{pH}$ values. At these $\mathrm{pH}$ values, half the ConA subunits exist in dimers, half in tetramers. The change in tetramer stability caused by osmolytes is qualitatively evident in the shifting of the $\mathrm{pH}$ midpoint. The increase in the $\mathrm{pH}$ midpoint of urea signals that the tetramer becomes unstable at a much higher $\mathrm{pH}$, thus the dimer is favored in the presence of urea. Conversely, the decrease in $\mathrm{pH}$ midpoint upon adding TMAO signals that the tetramer is stabilized at lower $\mathrm{pH}$. These results confirm the observation noted by Silvers and Myers that osmolytes can stabilize and destabilize not only protein secondary and tertiary structure but also quaternary structure. ${ }^{10}$ From this data, association constant values were calculated from Equation $2^{26}$ and used to construct the Van't Hoff plots shown in Figure 2. From the data in Figure 2, the free energy change, enthalpy change, and entropy change for the association of two dimers into a tetramer were determined, Table 1 . The association is endothermic and driven by entropy, as has been found previously. ${ }^{26,28}$ Without osmolytes, tetramers were present at higher temperatures $\left(\geq 40^{\circ} \mathrm{C}\right)$ over the $\mathrm{pH}$ range of 4.0 to 7.5 with little dissociation as recorded by CD measurements, while only dimeric ConA was present at low temperatures, despite $\mathrm{pH}$ changes.

\section{DISCUSSION}

Urea clearly increased both $\Delta \mathrm{H}$ and $\Delta \mathrm{S}$, while TMAO decreased both, Table 1 . These changes can be interpreted by considering the effect of the osmolytes on the hydration of the dimer-dimer interface. There are two main contributors to the $\Delta H$ of association. One, a negative contribution from the new interactions formed between the subunits. Two, a positive contribution from the interactions broken between the protein and the water-and other solutes - that were occupying the surface of the interface. Likewise, there are two main contributors to the $\Delta \mathrm{S}$ of association. One, a negative contribution from the organization of multiple subunits into a smaller number of oligomers and the fixing of side chains in the interface. Two, a positive contribution caused by the release of water-and other solutes-from the interface region. In the case of ConA, the solvent contribution to both $\Delta \mathrm{H}$ and $\Delta \mathrm{S}$ predominates. The association of the dimers is thus driven by the release of water from the interface region. Given the known effect of osmolytes on protein hydration, the alteration of thermodynamic parameters is not unexpected.

The change in enthalpy can be explained by considering the influence of urea and TMAO on the hydration of the dimer-dimer interface. It was suggested in Senear and Teller that the large and positive enthalpy of association was due to the steric hindrance around the Gln-122 residue, which is disordered in the tetramer. ${ }^{26}$ This appears less likely because of the impossibility of interaction of urea with the backbone of the residue that was stated as a mechanism for how urea destabilizes protein structure in Street et al. ${ }^{29}$ Instead, an alternate picture proposed by Huet and Claverie suggested that in the association of two dimers, the water associated with the hydrophobic binding region would require energy to displace the hydrogen bonds from the protein surface to bond with the bulk solvent. ${ }^{28}$ This model appears much more attractive as it allows for incorporation of the preferential hydration hypothesis. The molecular picture explains that when water transitions from bonding with the binding region to the bulk solvent, the change in bond energies results in the enthalpy. TMAO is preferentially excluded from the molecular surface, so water will accumulate around the protein under high concentrations of TMAO. A water mediated effect on enthalpy was also suggested in a study that found that there was no difference in enthalpy associated with TMAO stabilization of poly-alanine or poly-leucine chains. ${ }^{30}$ In comparison, urea will bind to the protein preferentially, mainly by hydrogen bonding to the peptide backbone, so its removal to the water poses a larger cost in enthalpy. A synthesis of the two competing theories could be a two stage model of protein-self assembly, similar to the model Chong and Ham proposed for $\beta$-amyloid formation in Alzheimer's. ${ }^{31}$ The initial, long-range attraction is mediated by the enthalpy driven hydration force of hydrophilic residues. After initial interaction, a short-range entropic force drives the association to completion by de-solvating the contact surfaces. This might explain the fact that urea increases the enthalpy change since more energy would be required to break the initial bond between the hydrophilic residue and urea, while still explaining the decreased enthalpy change seen in the TMAO results.

The preferential hydration model of osmolytes can similarly account for the change in entropy, Table 1. Urea, due to its tendency to interact with the protein backbone, localizes to the surface. Urea was theorized to act as a buffer that mitigates the entropic cost of exposing hydrophobic residues. ${ }^{3}$ This would enhance the stability of the dimer and the exposed hydrophobic dimer-dimer contact, reducing the need for water molecules to organize themselves to solvate the exposed interface. Conversely, TMAO is preferentially excluded from the protein surface and solvation of the dimer-dimer surface constitutes a larger entropic cost than solvation in the trials without osmolyte. ${ }^{5}$ Essentially, the alteration of $\Delta \mathrm{S}$ can be attributed to the relative increase in water ordering that occurs upon dissociation in TMAO and the relative decrease in water ordering that occurs upon dissociation in urea.

Valuable information may also be gained from examination of heat capacity change $\left(\Delta \mathrm{C}_{\mathrm{p}}\right)$. Although Senear and Teller were able to determine a value of $-800 \mathrm{cal} / \mathrm{mol} \bullet \mathrm{K}$, our data was collected, by necessity, over a fairly narrow temperature range, and attempts to accurately fit for $\Delta C_{p}$ were unsuccessful. A positive $\Delta C_{p}$ is common for protein unfolding according to Prabhu and Sharp, ${ }^{32}$ 
thus a negative value for self-association would be expected. The hydration of the protein is the major contributor to $\Delta \mathrm{C}_{\mathrm{p}},{ }^{32,33}$ thus a large variation upon addition of osmolytes would be expected since osmolytes change the water's interaction with the protein. It is anticipated that future work will address the effect of osmolytes on the heat capacity change of association.

\section{CONCLUSIONS}

The introduction of osmolytes like urea and TMAO causes a change in the association constant due to an increase in $\Delta H$ and $\Delta \mathrm{S}$ in the presence of urea and a decrease in the presence of TMAO. Our results are consistent with preferential hydration in TMAO solutions and preferential binding to urea in urea solutions. Previously, osmolytes were almost exclusively studied in the context of protein folding and not protein-protein interactions. To gain a more complete understanding of proteins in a biological context, where many form oligomers, it is necessary to understand how osmolytes influence the thermodynamics of protein protein interactions. While $\mathrm{pH}$, temperature, and salt ions are classically thought of as key environmental factors influencing protein binding, osmolytes may also have important effects on the thermodynamic parameters of protein-protein and protein ligand interactions.

\section{ACKNOWLEDGEMENTS}

The authors thank Connor Evins for carrying out some preliminary experiments, Lee Maiorano for technical assistance, and Davidson College for financial support of this research.

\section{REFERENCES}

1. Yancey, P. H., Clark, M. E., Hand, S. C., Bowlus, R. D., and Somero, G. N. (1982) Living with water stress: Evolution of osmolyte systems, Science 217, 1214-1222. DOI: 10.1126/science.7112124

2. Timasheff, S. N. (2001) Protein-solvent preferential interactions, protein hydration, and the modulation of biochemical reactions by solvent components, Proc Natl Acad Sci 99, 9721 - 9726. DOI: 10.1073/pnas.122225399

3. Adamczak, B., Wieczór, M., Kogut, M., Stangret, J., and Czub, J. (2016) Molecular basis of the osmolyte effect on protein stability: a lesson from the mechanical unfolding of lysozyme, Biochem J 473, 1 - 25. DOI: 10.1042/BCJ20160604

4. Schellman, J. A. (2003) Protein stability in mixed solvents: a balance of contact interaction and excluded volume, Biophys J 85, 108 - 125. DOI: 10.1016/S0006-3495(03)74459-2

5. Courtenay, E. S., Capp, M. W., Anderson, C. F., and Record, M. T., Jr. (2000) Vapor pressure osmometry studies of osmolyte-protein interactions: implications for the action of osmoprotectants in vivo and for the interpretation of "osmotic stress" experiments in vitro, Biochemistry 39, 4455 - 4471. DOI: 10.1021/bi9928871

6. Guinn, E. J., Pegram, L. M., Capp, M. W., Pollock, M. N., and Record, M. T., Jr. (2011) Quantifying why urea is a protein denaturant, whereas glycine betaine is a protein stabilizer. Proc Natl Acad Sci USA 108, 16932 - 16937. DOI: 10.1073/pnas.1109372108

7. Diehl, R. C., Guinn, E. J., Capp, M. W., Tsodikov, O. V., and Record, M. T., Jr. (2013) Quantifying additive interactions of the osmolyte proline with individual functional groups of proteins: comparisons with urea and glycine betaine, interpretation of m-values, Biochemistry 52, 5997 - 6010. DOI: 10.1021/bi400683y

8. Patel, C. N., Noble, S. M., Weatherly, G. T., Tripathy, A., Winzor, D. J., and Pielak, G. J. (2002) Effects of molecular crowding by saccharides on $\alpha$-chymotrypsin dimerization, Protein Sci 11, 997 - 1003. DOI: 10.1110/ps.4450102

9. Chebotareva, N. A., Andreeva, I.E., Makeeva, V. F., Livanova, N. B., and Kurganov, B. I. (2004) Effect of molecular crowding on self-association of phosphorylase kinase and its interaction with phosphorylase b and glycogen, J Mol Recognit 17 , 426 - 432. DOI: $10.1002 /$ jmr.696

10. Silvers, T. R., and Myers, J. K. (2013). Osmolyte effects on the self-association of concanavalin A: Testing theoretical models, Biochemistry 52, 9367 - 9374. DOI: 10.1021/bi401049s

11. Arakawa, T., and Timasheff, S. N. (1985) The stabilization of proteins by osmolytes, Biophys J 47, 411 - 414. DOI: 10.1016/S0006-3495(85)83932-1

12. Khan, S. H., Ahmad, N., Ahmad, F., and Kumar, R. (2010) Naturally occurring organic osmolytes: From cell physiology to disease prevention, IUBMB Life 62, 891 - 895. DOI: 10.1002/iub.406

13. Lin, T. Y., and Timasheff, A. N. (1994) Why do some organisms use a urea-methylamine mixture as osmolyte? Thermodynamic compensation of urea and trimethylamine N-oxide interactions with protein, Biochemistry 33, 12695 - 12701. DOI: $10.1021 / \mathrm{bi00208a021}$

14. Wang, A., Bolen, D. W. (1997) A naturally occurring protective system in urea-rich cells: Mechanism of osmolyte protection of proteins against urea denaturation, Biochemistry 36, 9101 - 9108. DOI: 10.1021/bi970247h 
15. Mello, C., and Barrick, D. (2003) Measuring the stability of partly folded proteins using TMAO, Protein Sci 12,1522 - 1529. DOI: $10.1110 /$ ps.0372903

16. Duff, M. R., and Howell, E. E. (2015) Thermodynamics and solvent linkage of macromolecule-ligand interactions, Methods 76, 51 - 60. DOI: 10.1016/j.ymeth.2014.11.009

17. Bergqvist, S., O’Brien, R., and Ladbury, J. E. (2001). Site-specific cation binding mediates TATA binding protein - DNA interaction from a hyperthermophilic archaeon, Biochemistry 40, $2419-2425$. DOI: 10.1021/bi002488m

18. Xavier, K. A., Shick, K. A., Smith-Gil, S. J., and Willson, R. C. (1997) Involvement of water molecules in the association of monoclonal antibody HyHEL-5 with bobwhite quail lysozyme, Biophys J 73, 2116 - 2125. DOI: $10.1016 /$ S00063495(97)78242-0

19. Olson, M. O. J., and Liener, I. E. (1967) Some physical and chemical properties of concanavalin a phytohemagglutinin of jack bean, Biochemistry 6, 105 - 111. DOI: 10.1021/bi00853a018

20. Agrawal, B. B., and Goldstein, I. J. (1968) Protein-carbohydrate interaction. VII. Physical and chemical studies on concanavalin A, the hemagglutinin of the jack bean, Arch Biochem Biophys 124, 218 - 229. DOI: 10.1016/0003-9861(68)903226

21. Hardman, K. D., and Ainswort, C. F. (1972) Structure of concanavalin A at 2.4 angstrom resolution, Biochemistry 11, $4910-$ 4919. DOI: $10.1021 /$ bi00776a006

22. Becker, J. W., Reeke, G. N., Jr, Wang, J. L., Cunningham, B. A., and Edelman, G. M. (1975) The covalent and threedimensional structure of concanavalin A. III. Structure of the monomer and its interactions with metals and saccharides, $J$ Biol Chem 250, 1513 - 1524.

23. Parkin, S., Rupp, B., and Hope, H. (1996) Atomic resolution structure of concanavalin A at 120 K, Acta Crystallogr, Sect D: Biol Crystallogr 52, 1161 - 1168. DOI: 10.1107/S0907444996009237

24. Brewer, C. F., Marcus, D. M., and Grollman, A. P. (1974) Interactions of saccharides with concanavalin: a relation between calcium ions and the binding of saccharides to concanavalin A, J Biol Chem 249, 4614 - 4616. DOI: 10.1021/bi00746a023

25. Goldstein, I. J., and Poretz, R. D. (1971) Protein-carbohydrate interaction: On the mode of binding of aromatic moieties to concanavalin A, Biochem Pharmacol 20, 2727 - 2739. DOI: 10.1016/0006-2952(71)90182-1

26. Senear, D. F., and Teller, D. C. (1981) Thermodynamics of concanavalin A dimer-tetramer self-association: sedimentation equilibrium studies, Biochemistry 20, 3076 - 3083. DOI: 10.1021/bi00514a014

27. Singh, R., Haque, I., and Ahmad, F. (2005) Counteracting osmolyte trimethylamine N-oxide destabilizes proteins at pH below its $\mathrm{p} K_{a}$ : Measurements of thermodynamic parameters of proteins in the presence and absence of trimethylamine $N$ oxide, J Biol Chem 280, 11035 - 11042. DOI: 10.1074/jbc.M410716200

28. Huet, M., Claverie, J. M. (1978) Sedimentation studies of the reversible dimer-tetramer transition kinetics of concanavalin A, Biochemistry 17, 236 - 241. DOI: 10.1021/bi00595a007

29. Street, T. O.; Bolen, D. W.; Rose, G. D. (2006) A molecular mechanism for osmolyte-induced protein stability. Proc Natl Acad Sci 103, 13997 - 14002. DOI: 10.1073/pnas.0606236103

30. Su, Z., Srinivasa, J. R., and Dias, C. L. (2015) Roles of urea and TMAO on the interaction between extended non-polar peptides, Biophys J 108, 519 - 520. DOI: 10.1016/j.bpj.2014.11.2849

31. Chong, S. H., and Ham, S. (2012) Impact of chemical heterogeneity on protein self-assembly in water, Proc Acad Natl Sci 109 , 7636 - 7641. DOI: $10.1073 /$ pnas.1120646109

32. Prabhu, N. V., Sharp, K. A. (2005) Heat capacity in proteins, Annu Rev Phys Chem 56, 521 - 548. DOI: 10.1146/annurev.physchem.56.092503.141202

33. Sturtevant, J. M. (1977) Heat capacity and entropy changes in processes involving proteins, Proc Acad Natl Sci 74, 22362240.

\section{ABOUT THE STUDENT AUTHORS}

Tyler Pfister graduated from Davidson College in 2017 with a degree in chemistry and emphasis in biological chemistry. He is now a research fellow at the National Institute of Health and plans to pursue a MD/PhD. Shamus Cooper graduated from Davidson College in 2016 with a degree in chemistry and emphasis in biological chemistry. He will be applying to dental programs.

\section{PRESS SUMMARY}

To understand how proteins interact with each other in cells, we studied how proteins separate in the presence of osmolytes. These small organic compounds are produced in many different organisms, where they help to mitigate water stress. We found that it takes less energy to separate proteins when urea, an osmolyte filtered out of your body into urine, is included. Conversely, TMAO, an osmolyte found in fish, causes an increase in the energy required to separate proteins. These results will help us understand how the cellular environment can influence the interactions of proteins with each other, a process that is fundamental in the progression of several neurodegenerative diseases like Alzheimer's and Parkinson's Disease. 